International Journal of Engineering \& Technology, $7(2.8)(2018) 204-210$
International Journal of Engineering \& Technology
WPC
Website: www.sciencepubco.com/index.php/IJET
Research Paper

\title{
Performance analysis of number theoretic transform-based convolution using field programmable gate array
}

\author{
G.A.E Satish Kumar \\ Professor, Vardhaman College of Engineering, Shamshabad, Hyderabad, \\ Email: gaesathi@gmail.com
}

\begin{abstract}
This paper presents the convolution operation based on the Number Theoretic Transfom for two $n=8$ input sequences. The convolution of two n-point sequences using Fast Fourier Transform exhibits design complexity leading to high power consumption. The Number Theoretic Transform utilizes the matrix of modulus values to evaluate the convolution. The Number Theoretic Transform is as an integer transform which makes the design comparatively simple. The convolution based Number Theoretic Transform is developed using the Very High Speed Integrated Circuit Hardware Description language.Also the real time implementation of the proposed method is validated by the Xilinx Spartan FPGA family devices. The performance analysis of power, speed and area are evaluated and compared with 3A DSP FPGA and Virtex 6 FPGA devices.
\end{abstract}

Keywords: Convolution, Number Theoretic Transform, VHDL, Xilinx Spartan FPGA

\section{Introduction}

The convolution is a mathematical operation between two functions to generate the third function. Depending on the type of signal, the convolution can be classified as discrete and analog. The discrete convolution is used more predominantly compared to the analog convolution due to high precision, high performance and easy debugging. The convolution is classified as linear and circular convolution depending on the periodicity of the signal.Gradually, the circular convolution got evolved for its faster operation. The circular convolution uses finite number of inputs in operations. The convolution could be performed by several methods like sectioned convolution, graphical convolution, fast fourier transform and vedic multiplication algorithm[1].

Modern day applications such as signal and image processing, acoustics, electrical engineering demand faster convolution algorithm. By making use of Fast Fourier Transform (FFT), the convolution is converted to ordinary multiplication of the input sequence. As the FFT includes complexity in the design and high power consumption, the Number Theoretic Transform (NTT) is utilized.

The Number Theoretic Transform (NTT) is a finite integer convolution algorithm. In NTT, the complex FFT algorithms with the twiddle factors are replaced with the modulus operation. The NTT can be effectively used in the lossless medical image transmission by water marking approach [2]. The NTT's effectiveness has been proven in the images lossless transmission and in convolution fast calculation [3] The NTT combined with lattice based cryptosystems performs cyclic, negacyclic and convolutions in encrypted domain[4].NTT algorithm are improvised for the float value transforms for Fourier, Hartley, Sine and Cosine signals [5]

This paper proposes the design of convolution based on NTT algorithm using the VHDL coding. The VHDL coding of the FFT based convolution is time consuming in design and occupies more number of multipliers and adders. By utilizing the NTT algorithm, the VHDL design is simple and thus easily downloadable in the FPGAs. The FPGA more advantages than other digital controllers in high speed operation, low power consumption, parallel processing and reconfigurable design. The convolution neural network based on FPGA is effectively used in image identification [6].The FPGA accelerator for the 3D convolution design aides to avoid the loading repetition of the processing feature maps[7]. The performance of deep convolution neural network is 1.9 to 250 times faster by utilizing FPGA device[2][8]. Controllers analyses for nonlinear systems has been reported [9-18]

The real time implementation of the convolution based on NTT algorithmis evaluated by using the FPGA devices namely Xilinx Spartan 3A DSP FPGA and Xilinx Virtex 6 FPGA. The next section discuss on the convolution based NTT algorithmpreceded by the FPGA based design flow of the proposed method.

\section{The Proposed Convolution Method}

The convolution of two sequences of length $\mathrm{N}=8$ is performed using the NTT algorithm. The NTT algorithm uses the modulus function for the evaluation of the FFT.The procedure for the NTT algorithm is as follows.

i. The order of the sequence (n) for the FFT is specifies as nonnegative integer.

ii. The modulus $\mathrm{M}$ is chosen such that every value of the input sequence is within range of 0 to $M$. (i.e.,) $1 \leq n \leq M$.

iii. The formula for the working module in the NTT algorithm is given by

iv.

$\mathrm{N}=\mathrm{kn}+1$

Where $\mathrm{k}$ is an integer $>1$,

$\mathrm{n}$ is the order of the sequence. 
Note: The $\mathrm{N}$ value generated using this formula should be a prime number using the Dirichlet's Theorem.

v. For the n-point DFT, $\omega$ is the primitive $\mathrm{n}^{\text {th }}$ root of unity. This is compensated in the NTT algorithm by using Euler's Theorem defined as vi.

$\omega=\mathrm{g}^{\mathrm{k}} \bmod \mathrm{N}$,

Where $\mathrm{g}$ is the generator

The value of the generator " $g$ " is selected by using following conditions

a) The value of $g$ is assumed to be say a.

b) The prime number $\mathrm{N}$ is considered as $\mathrm{N}-1$ and factorise as two product values say $\mathrm{x}$ and $\mathrm{y}$.

c) Now the generator "a" is found bychecking for the following d) $\mathrm{a}^{\mathrm{N}-1} \bmod \mathrm{M}=1$

$\mathrm{a}^{\mathrm{x}} \bmod \mathrm{M} \neq 1$ and $\mathrm{a}^{\mathrm{y}} \bmod \mathrm{M} \neq 1$

vii. After finalising with the values of $\mathrm{N}, \mathrm{M}$ and $\mathrm{a}$; the convolution based on NNT algorithm is evaluated

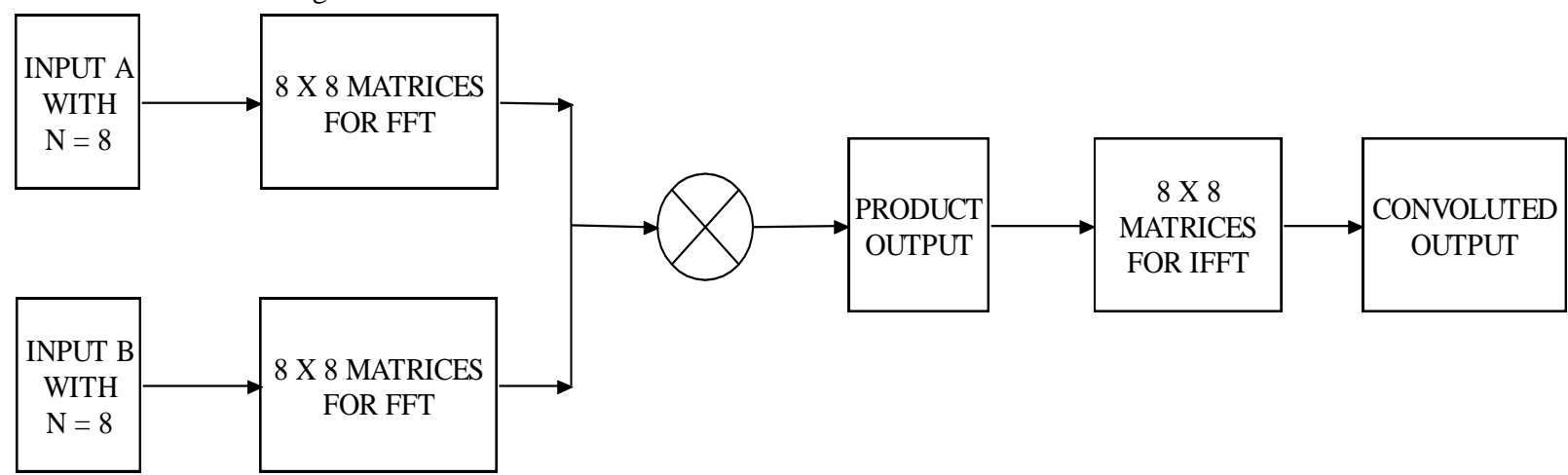

Fig. 1: Block diagram of the Number Theoretic Transform with sequence $n=8$

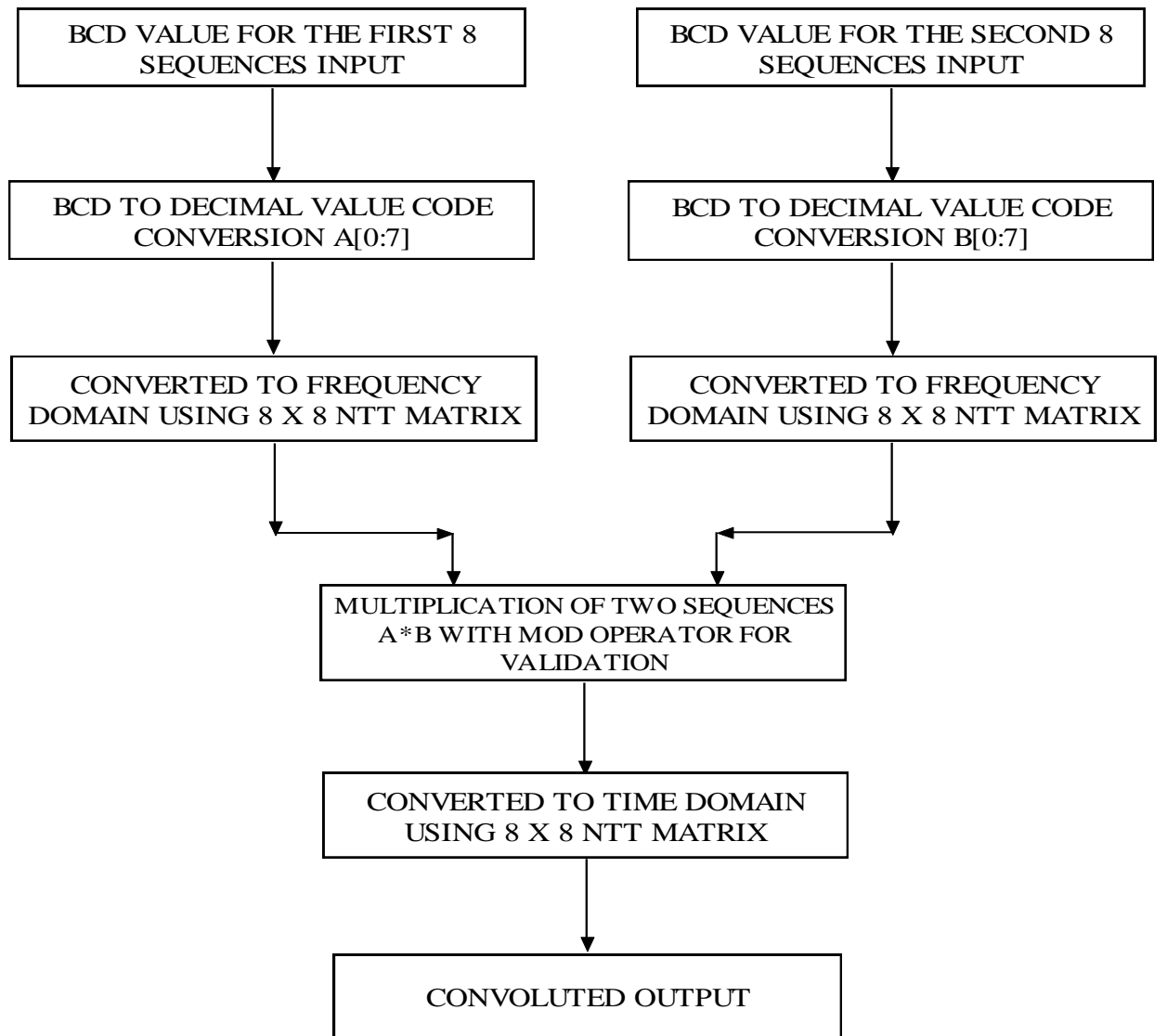

Fig. 2: FPGA Design flow of the propose convolution method viii. The two $n$ sequences are fed in through the $8 \mathrm{X} 8$ matrices. The $8 \mathrm{X} 8$ matrices of the NTT algorithm is given below

$$
\left(\begin{array}{ccc}
\left(W_{8}^{0}\right)^{0} & \cdots & \left(W_{8}^{0}\right)^{7} \\
\vdots & \ddots & \vdots \\
\left(W_{8}^{7}\right)^{0} & \cdots & \left(W_{8}^{7}\right)^{7}
\end{array}\right)
$$

vii. The FFT manipulated values are multiplied for the evaluation of the 8 point values which is again fed the IFFT based NTT algorithm using the following $8 \mathrm{X} 8$ matrices as shown below

$\left(\begin{array}{ccc}\left(W_{8}^{0}\right)^{-0} & \cdots & \left(W_{8}^{0}\right)^{-7} \\ \vdots & \ddots & \vdots \\ \left(W_{8}^{7}\right)^{-0} & \cdots & \left(W_{8}^{7}\right)^{-7}\end{array}\right)$

vii. The convoluted output of the 8 point input sequences are viii. The block diagram for the proposed NTT method is shown in Fig.1 
The FPGA design flow of the proposed method is depicted in Fig.2.The inputs are fed in the BCD format, as the inputs could be assigned to the IO pins in real time FPGA implementation. The $\mathrm{BCD}$ of the two 8 sequences are converted into the integer values using the $\mathrm{BCD}$ to decimal code converter. The two 8 integer values are converted to frequency domain equivalence by using the $8 \mathrm{X} 8$ NTT matrices. The NTT matrix is framed considering the modulus function as equivalent to the twiddle factors of the FFT algorithm. The two frequency converted 8 sequences are multiplied and operated with the mod operator to make sure that the value is not greater than the value of modulus value $\mathrm{M}$. The product obtained has to be converted back to time domain using INTT matrix. The INTT is performed on the obtained 8 integer value using the $8 \mathrm{X} 8$ NNT inverse matrices multiplied with the $\mathrm{n}^{-1} \bmod \mathrm{M}$ to yield the desired output. The VHDL code of the proposed convolution method involves the structure style of modelling.

\section{Results and Discussion}

In this work, the inputs values for the two input sequences are verified with three different combinations. The prime number 673 is fixed as the modulus value ' $M$ '. The generator value is assumed as $\mathrm{W}=326$. The first set of two inputs say $\mathrm{x}=\{4,1,4,2,1,3,5,6\}$ and $y=\{6,1,8,0,3,3,9,8\}$ assigned to the proposed convolution based NTT algorithm gives the result of convolution of $\left(x^{*} \mathrm{y}\right)=\{123,120,106,92,139,144,140,124\}$. The second set of two inputs are assigned as $x=\{4,3,2,1,0,0,0,0\}$ and $y=\{8,7,6,5,0,0,0,0\}$ to given the convoluted result as $\{32,52,61,60,34,16,5,0\}$. Similarly for the third set of two inputs $\mathrm{x}=\{1,1,2,2,3,3,4,4\}$ and $y=\{5,5,6,6,7,7,8,8\}$, the convoluted output is given as $\{126,132,134,136,134,132,126,120\}$. Fig. 3 shows the simulated output for the three different input sets using the proposed convolution based on NTT algorithm using the ModelSim software. The real time implementation of the proposed method is performed using the Xilinx Spartan family devices like 3A DSP FPGA and Virtex 6 FPGA. The RTL view with the detailed schematic of the propose method in depicted in the Fig.4.The area analysis of the proposed method using Xilinx Spartan 3A DSP and Virtex 6 FPGA are presented in Table $1 \& 2$ respectively. The area utilization of the proposed method is low for the Virtex 6 FPGA. The performance of the power holds good for the Xilinx Spartan 3A DSP FPGA as proven by comparing the Tables $3 \& 4$. The timing analysis of the proposed method using the FPGA is presented in Table 5.

\begin{tabular}{|c|c|c|c|c|}
\hline BCD INPUTS & & & & \\
\hline 田-1/ntt_fft_updated/nt11 & 0100 & 0100 & & 0001 \\
\hline 田-1/ntt_fft_updated/nt12 & 0001 & 0001 & S0011 & S0001 \\
\hline +1-1/ntt_fft_updated/nt13 & 0100 & 0100 & 0010 & \\
\hline 田-1 /ntt_fft_updated/nt14 & 0010 & 0010 & 0001 & $\sqrt{0010}$ \\
\hline +1-1/ntt_fft_updated/nt15 & 0001 & 0001 & $\sqrt{0000}$ & 0011 \\
\hline 田-1/ntt_fft_updated/nt16 & 0011 & 0011 & 90000 & 80011 \\
\hline 田-U/ntt_fft_updated/nt17 & 0101 & 0101 & $\sqrt{0000}$ & $\sqrt{0100}$ \\
\hline 田-1/ntt_fft_updated/nt18 & 0110 & 0110 & 90000 & 90100 \\
\hline 田-J /ntt_fft_updated/nt21 & 0110 & 01110 & $\sqrt{1000}$ & $\sqrt{0101}$ \\
\hline 田-1/ntt_fft_updated/nt22 & 0001 & 0001 & 0111 & 80101 \\
\hline 田-1/ntt_fft_updated/nt23 & 1000 & 1000 & 90110 & \\
\hline 田-1/ntt_fft_updated/nt24 & 0000 & 0000 & S0101 & $\sqrt{0110}$ \\
\hline 田-1/ntt_fft_updated/nt25 & 0011 & 0011 & 90000 & 0111 \\
\hline 田-1/ntt_fft_updated/nt26 & 0011 & 0011 & 80000 & 0111 \\
\hline 田-1/ntt_fft_updated/nt27 & 1001 & 1001 & 30000 & 1000 \\
\hline 田-1/ntt_fft_updated/nt28 & 1000 & 1000 & 30000 & $\$ 1000$ \\
\hline \multicolumn{5}{|l|}{ OUTPUT } \\
\hline I/ntt_fft_updated/n1 & 123 & 123 & $\sqrt{32}$ & 126 \\
\hline $1 /$ ntt_fft_updated/n2 & 120 & 120 & 52 & 132 \\
\hline I/ntt_fft_updated/n3 & 106 & 106 & 61 & 134 \\
\hline I/ntt_fft_updated/n4 & 92 & 92 & 160 & 136 \\
\hline $1 /$ ntt_fft_updated/n5 & 139 & 139 & 34 & 134 \\
\hline $1 /$ ntt_fft_updated $/ \mathrm{n} 6$ & 144 & 144 & $\sqrt{16}$ & 132 \\
\hline $1 /$ ntt_fft_updated $/ \mathrm{n} 7$ & 140 & 140 & $\sqrt{5}$ & $\sqrt{126}$ \\
\hline I /ntt_fft_updated/n8 & 124 & 124 & 10 & $\sqrt{120}$ \\
\hline \multicolumn{5}{|c|}{ INTEGER CONVERTED INPUTS } \\
\hline I/ntt_fft_updated/a1 & 4 & 4 & & 1 \\
\hline I/ntt_fft_updated/a2 & 1 & 1 & $\sqrt{3}$ & 1 \\
\hline I/ntt_fft_updated/a3 & 4 & 4 & $\sqrt{2}$ & \\
\hline $1 /$ intt_fft_updated/a4 & 2 & 2 & 11 & $\sqrt{2}$ \\
\hline 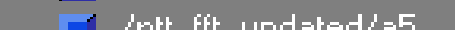 & & & 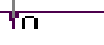 & T2 \\
\hline
\end{tabular}

Fig. 3: Simulation output of the NTT algorithm using the MODELSIM software 


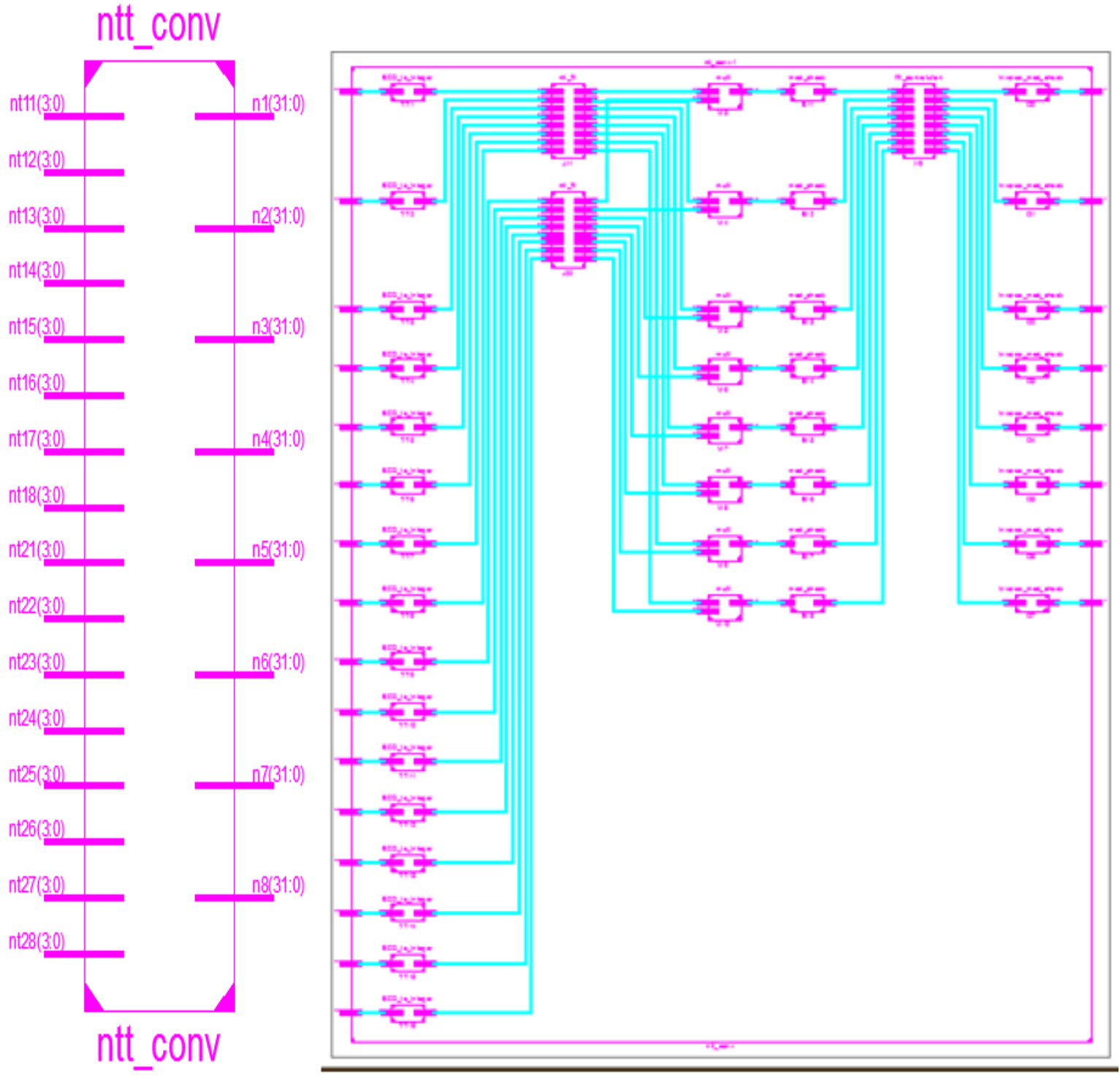

Fig. 4: RTL view with detailed schematic view of the proposed convolution based on NTT algorithm using the Xilinx Spartan FPGA device

Table 1: Device Utilization chart of the propose method using the Xilinx Spartan 3A DSP FPGA

\begin{tabular}{|c|c|c|c|}
\hline \multicolumn{2}{|c|}{ Device Utilization Summary } & Available & Utilization \\
\hline Logic Utilization & 64 & 33,280 & $1 \%$ \\
\hline Number of Slice Latches & 1,806 & 33,280 & $5 \%$ \\
\hline Number of 4 input LUTs & 1,080 & 16,640 & $6 \%$ \\
\hline Number of occupied Slices & 1,080 & 1,080 & $100 \%$ \\
\hline Total Number of 4 input LUTs & 1,900 & 33,280 & $5 \%$ \\
\hline Number used as logic & 1,806 & & \\
\hline Number used as a route-thru & 94 & & \\
\hline Number of bonded IOBs & 320 & 519 & $61 \%$ \\
\hline Number of DSP48As & 40 & 84 & $47 \%$ \\
\hline Average Fanout of Non-Clock Nets & 1.86 & & \\
\hline
\end{tabular}


Table 2: Device Utilization chart of the propose method using the Xilinx Virtex 6 FPGA

\begin{tabular}{|c|c|c|c|}
\hline \multicolumn{4}{|c|}{ Device Utilization Summary } \\
\hline Slice Logic Utilization & Used & Available & Utilization \\
\hline Number of Slice Registers & 190 & 301,440 & $1 \%$ \\
\hline Number of Slice LUTs & 1,691 & 150,720 & $1 \%$ \\
\hline Number of occupied Slices & 630 & 37,680 & $1 \%$ \\
\hline Number of LUT Flip Flop pairs used & 1,691 & & \\
\hline Number with an unused Flip Flop & 1,501 & 1,691 & $88 \%$ \\
\hline Number with an unused LUT & 0 & 1,691 & $0 \%$ \\
\hline Number of fully used LUT-FF pairs & 190 & 1,691 & $11 \%$ \\
\hline Number of unique control sets & 64 & & \\
\hline $\begin{array}{c}\text { Number of slice register sites lost } \\
\text { to control set restrictions }\end{array}$ & 448 & 301,440 & $1 \%$ \\
\hline Number of bonded IOBs & 320 & 600 & $53 \%$ \\
\hline Number of DSP48E1s & 36 & 768 & $4 \%$ \\
\hline Average Fanout of Non-Clock Nets & 2.29 & & \\
\hline
\end{tabular}

Table 3: Power Analysis of the proposed method using the Xilinx Spartan 3A DSP FPGA

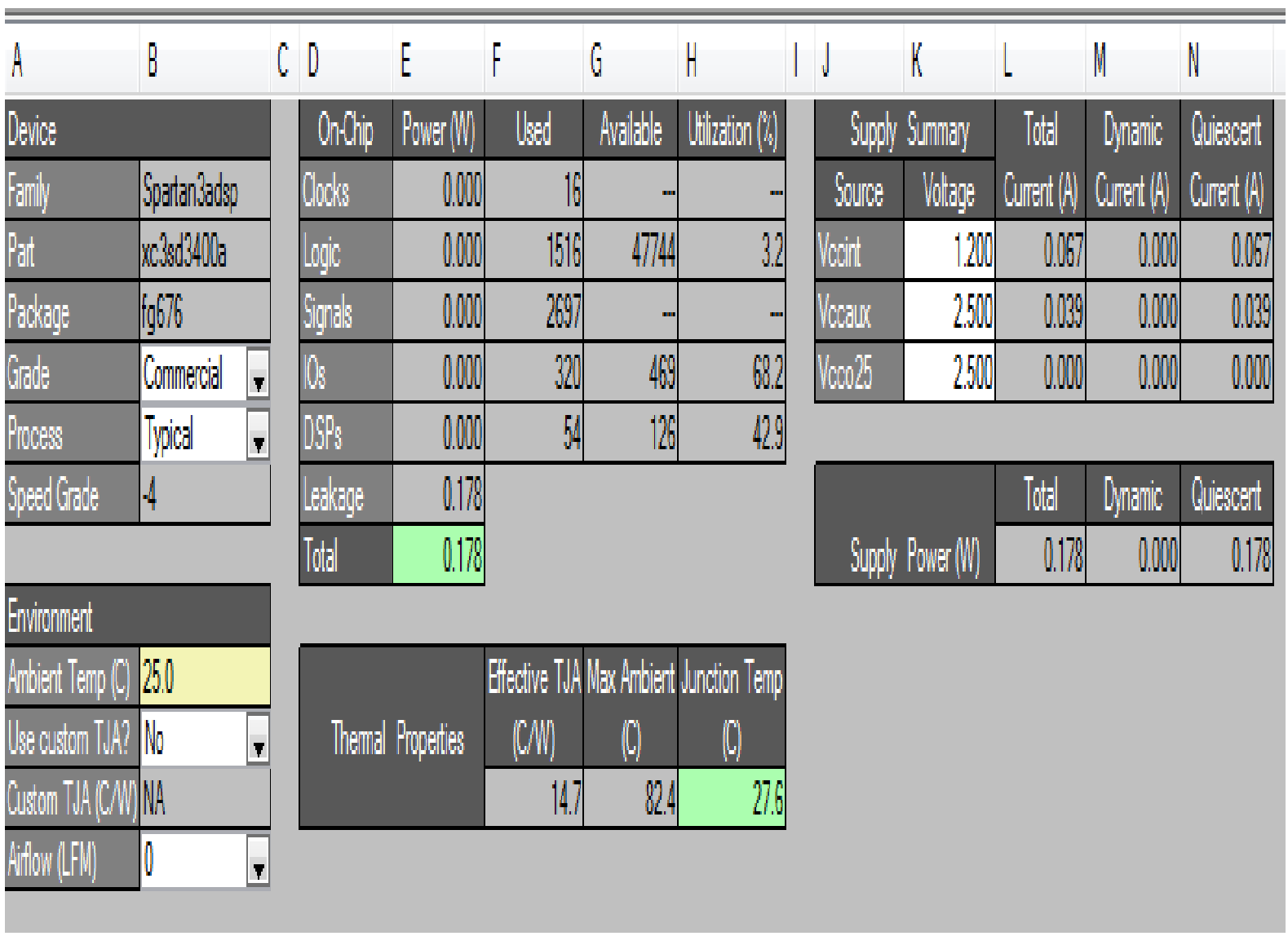

Table 4: Power Analysis of the proposed method using the Xilinx Virtex 6 FPGA 


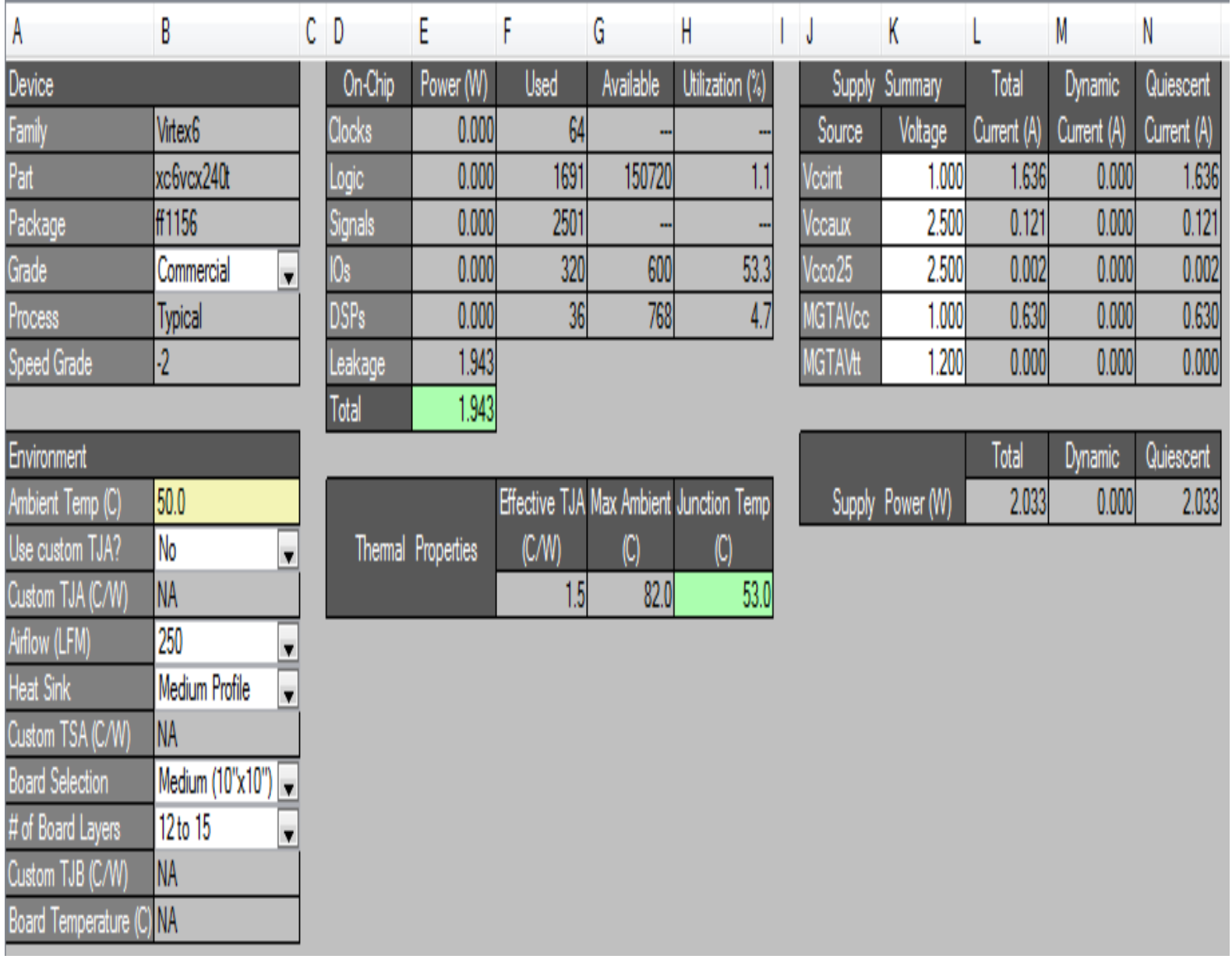

Table 5: Timing Analysis of the proposed method using the Xilinx FPGA devices

\begin{tabular}{|c|c|c|}
\hline Methods & SPARTAN 3A DSP & VIRTEX 6 \\
\hline Max Delay & $2.852 \mathrm{~ns}$ & $1.214 \mathrm{~ns}$ \\
\hline Number of paths & 10 & 8 \\
\hline Number of destination ports & 4 & 1 \\
\hline Memory Utilized & $306696 \mathrm{~KB}$ & $350460 \mathrm{~KB}$ \\
\hline Total Real Time to MAP & $6 \mathrm{sec}$ & $13 \mathrm{sec}$ \\
\hline Total Real Time to PAR & $1 \mathrm{~min} 10 \mathrm{sec}$ & $1 \mathrm{~min} 35 \mathrm{sec}$ \\
\hline
\end{tabular}

\section{Conclusions}

The design of convolution based NTT is developed and executed using the VHDL coding. The real time validation using the FPGA for the proposed method seems to be satisfactory. The power and area hold good for the Xilinx Spartan 3A DSP FPGA. The Xilinx Virtex 6 FPGA proves to be fast in operation with the maximum delay of $1.214 \mathrm{~ns}$. The extension of this work could be performed with the floating point multiplication using NTT algorithm.

\section{References}

[1] R. Nagaraju, T. Chandra Prakash, A. Venkateshwarlu, "A Nove High Speed Convolution and De-convolutionAlgorithm Implementation Based on Ancient Indian Vedic Mathematics", International Journal of VLSI systems and Communication systems, vol.3, no.4, July 2015, pp: 514-517.

[2] RaghidMorcel, MazenEzzeddine, HaithamAkkary, "FPGABased Accelerator for Deep Convolutional Neural Networks for the SPARK Environment", 2016 IEEE International Conference on Smart Cloud (SmartCloud), Nov 2016, DOI 10.1109/SmartCloud.2016.31.

[3] LamriLaouamer, "Towards a robust and fully reversible image watermarking framework based on number theoretic transform", International Journal of Signal and Imaging Systems Engineering, vol.10, no.4, April 2017, pp.169 - 177
[4] Alberto Pedrouzo-Ulloa, Juan RamºnTroncoso-Pastoriza, and Fernando Pérez-González, "Number Theoretic Transforms for SecureSignal Processing" , IEEE Transactions on Information Forensics And Security, vol. 12, no. 5, May 2017, pp: 1125-1140.

[5] Paulo Hugo E. S. Lima, Juliano B. Lima and Ricardo M. Campello de Souza, "Fractional Fourier, Hartley, Cosine and Sine Number-Theoretic Transforms Based on Matrix Functions", Circuits, Systems, and Signal Processing, vol.36, no.7, July 2017, pp 2893-2916.

[6] CongyiLyu, Haoyao Chen, Xin Jiang, Peng Liand Yunhui Liu, "Real-time object tracking system basedon field-programmable gate arrayand convolution neural network", International Journal of AdvancedRobotic Systems,Special Issue,Feb 2017, pp:1-14.

[7] Hai Wang, Mengjun Shao, Yan Liu, and Wei Zhao, "Enhanced Efficiency 3D Convolution Based on Optimal FPGA Accelerator", IEEE Access, vol.5, April 2017, pp: 6909-6916

[8] Mohammad Motamedi ,Philipp Gysel, and VenkateshAkella"Design space exploration of FPGA-based Deep Convolutional Neural Networks",Design Automation Conference (ASP-DAC), 2016 21st Asia and South Pacific, Mar 2016, DOI: 10.1109/ASPDAC.2016.7428073.

[9] R. Kalaivani, K. Ramash Kumar, S. Jeevananthan, "Implementation of VSBSMC plus PDIC for Fundamental Positive Output Super Lift-Luo Converter,” Journal of Electrical Engineering, Vol. 16, Edition: 4, 2016, pp. 243-258.

[10] K. Ramash Kumar,'Implementation of Sliding Mode Controller plus Proportional Integral Controller for Negative Output Elementary Boost Converter," Alexandria Engineering Journal (Elsevier), 2016, Vol. 55, No. 2, pp. 1429-1445.

[11] P. Sivakumar, V. Rajasekaran, K. Ramash Kumar, "Investigation of Intelligent Controllers for Varibale Speeed PFC Buck-Boost Rectifier Fed BLDC Motor Drive," Journal of Electrical Engineering (Romania), Vol.17, No.4, 2017, pp. 459-471.

[12] K. Ramash Kumar, D.Kalyankumar, DR.V.Kirbakaran" An Hybrid Multi level Inverter Based DSTATCOM Control, Majlesi Journal of Electrical Engineering, Vol. 5. No. 2, pp. 17-22, June 2011, ISSN: 0000-0388.

[13] K. Ramash Kumar, S. Jeevananthan, "A Sliding Mode Control for Positive Output Elementary Luo Converter,” Journal of Electrical Engineering, Volume 10/4, December 2010, pp. 115-127. 
[14] K. Ramash Kumar, Dr.S. Jeevananthan,” Design of a Hybrid Posicast Control for a DC-DC Boost Converter Operated in Continuous Conduction Mode" (IEEE-conference PROCEEDINGS OF ICETECT 2011), pp-240-248, 978-1-42447925-2/11.

[15] K. Ramash Kumar, Dr. S. Jeevananthan,” Design of Sliding Mode Control for Negative Output Elementary Super Lift Luo Converter Operated in Continuous Conduction Mode", (IEEE conference Proceeding of ICCCCT-2010), pp. 138-148, 978-14244-7768-5/10.

[16] K. Ramash Kumar, S. Jeevananthan, S. Ramamurthy" Improved Performance of the Positive Output Elementary Split InductorType Boost Converter using Sliding Mode Controller plus Fuzzy Logic Controller, WSEAS TRANSACTIONS on SYSTEMS and CONTROL, Volume 9, 2014, pp. 215-228.

[17] N. Arunkumar, T.S. Sivakumaran, K. Ramash Kumar, S. Saranya, "Reduced Order Linear Quadratic Regulator plus Proportional Double Integral Based Controller for a Positive Output Elementary Super Lift Luo-Converter," JOURNAL OF THEORETICAL AND APPLIED INFORMATION TECHNOLOGY, July 2014. Vol. 65 No.3, pp. 890-901.

[18] Arunkumar, T.S. Sivakumaran, K. Ramash Kumar, "Improved Performance of Linear Quadratic Regulator plus Fuzzy Logic Controller for Positive Output Super Lift Luo-Converter," Journal of Electrical Engineering, Vol. 16, Edition:3, 2016, pp. 397-408.

[19] N Bala Dastagiri, K Hari Kishore "Novel Design of Low Power Latch Comparator in $45 \mathrm{~nm}$ for Cardiac Signal Monitoring", International Journal of Control Theory and Applications, ISSN No: 0974-5572, Vol No.9, Issue No.49, page: 117-123, May 2016.

[20] T. Padmapriya and V. Saminadan, "Distributed Load Balancing for Multiuser Multi-class Traffic in MIMO LTE-Advanced Networks", Research Journal of Applied Sciences, Engineering and Technology (RJASET) - Maxwell Scientific Organization, ISSN: 2040-7459; e-ISSN: 2040-7467, vol.12, no.8, pp: 813-822, April 2016.

[21] S.V.Manikanthan and V.Rama "Optimal Performance of Key Predistribution Protocol In Wireless Sensor Networks" International Innovative Research Journal of Engineering and Technology, ISSN NO: 2456-1983, Vol-2, Issue -Special-March 2017.

[22] S.V.Manikanthan and K.Baskaran "Low Cost VLSI Design Implementation of Sorting Network for ACSFD in Wireless Sensor Network", CiiT International Journal of Programmable Device Circuits and Systems,Print: ISSN 0974 - 973X \& Online: ISSN 0974 - 9624, Issue : November 2011, PDCS112011008.

[23] K. Ramash Kumar, Dr. S. Jeevananthan,” Design of Sliding Mode Control for Negative Output Elementary Super Lift Luo Converter Operated in Continuous Conduction Mode", (IEEE conference Proceeding of ICCCCT-2010), pp. 138-148, 978-14244-7768-5/10. 